\title{
Measurements of top quark properties with the ATLAS detector
}

\author{
Christopher Unverdorben ${ }^{1, a}$, on behalf of the ATLAS Collaboration \\ ${ }^{1}$ Am Coulombwall 1, 85748 Garching, Germany
}

\begin{abstract}
A selection of recent top quark measurements performed with the ATLAS detector is presented here, with data taken in 2011 and 2012 at centre-of-mass energies of $7 \mathrm{TeV}$ and $8 \mathrm{TeV}$, respectively. The analyses cover the determination of the top pole mass by a cross-section measurement, the top/anti-top quark mass difference, the spin correlation and the charge asymmetry. No hints for physics beyond the Standard Model have been found but a great reduction of uncertainties compared to former results was achieved.
\end{abstract}

\section{Introduction}

The top quark is the heaviest elementary particle known today and hence has a strong connection to the Higgs particle and the vacuum stability. Precision studies testing the physics of the Standard Model (SM) and beyond are therefore driven by top quark measurements. In addition, the top lifetime $\tau_{\text {top }} \approx 0.5 \times 10^{-25} \mathrm{~s}[1]$ is very short which does not allow hadronisation before its decay, giving the opportunity to study a bare quark with its initial properties.

The advantage of the ATLAS experiment [2] compared to former experiments, is the high amount of top quarks produced in proton-proton collisions at the LHC. The production channel is dominated by the strong interaction and the main process is the gluon-gluon fusion.

\section{Determination of the top pole mass via a cross-section measurement}

By measuring the $t \bar{t}$ cross-section $\sigma_{t \bar{t}}$ with high precision, theory allows a determination of the top pole mass $m_{t}^{\text {pole }}$ [3]. This approach is predicted to differ from a direct measurement of the top quark mass by only $O(1 \mathrm{GeV})[4]$ and is therefore very interesting for a comparison with that.

The determination of the required cross-section was performed with data from $2011(\sqrt{s}=7 \mathrm{TeV})$ and $2012(\sqrt{s}=8 \mathrm{TeV})$, having the integrated luminosities of $4.6 \mathrm{fb}^{-1}$ and $20.3 \mathrm{fb}^{-1}$, respectively. All events were reconstructed in the di-leptonic $e \mu$ channel with one $\mathrm{W}$ boson decaying to electron + antineutrino ${ }^{2}$ and the other to muon + anti-neutrino. The dataset was separated into two by distinguishing events with exactly one $b$-quark ( $b$-tagged jet) from those with exactly two. The total numbers of events per dataset were described by two independent equations, whereas two parameters remained unknown: the cross-section $\sigma_{t \bar{t}}$ and the efficiency to reconstruct $b$-tagged jets $\varepsilon_{b}$.

\footnotetext{
ae-mail: Christopher.Unverdorben@physik.uni-muenchen.de

${ }^{2}$ Charge conjugations are always implied in this article.
} 


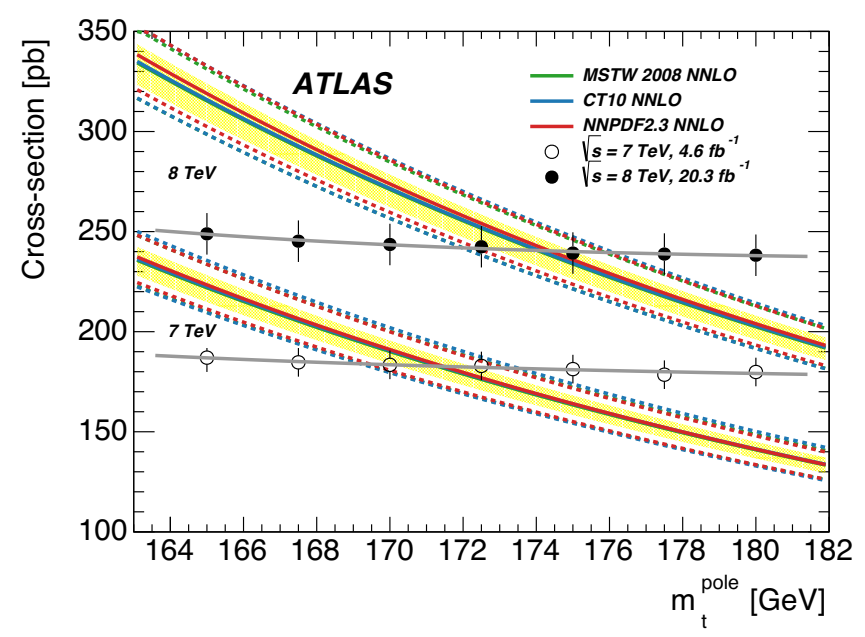

Figure 1: Lines represent the theoretical predicted cross-sections as a function of $m_{t}^{\text {pole }}$, circles the measured values of $\sigma_{t \bar{t}}$ with dependencies on $m_{t}$ used in the simulations. The intersection points lead to the values of $m_{t}^{\text {pole }}$. Upper part for $\sqrt{s}=8 \mathrm{TeV}$, lower part for $\sqrt{s}=7 \mathrm{TeV}$ [3].

$$
\begin{aligned}
& N_{1}=L \sigma_{t \bar{t}} \varepsilon_{e \mu} 2 \varepsilon_{b}\left(1-C_{b} \varepsilon_{b}\right)+N_{1}^{b k g d} \\
& N_{2}=L \sigma_{t \bar{t}} \varepsilon_{e \mu} C_{b} \varepsilon_{b}^{2}+N_{2}^{b k g d}
\end{aligned}
$$

for $1 b$-tagged jet

for $2 b$-tagged jets

Here $N$ is the number of events, $N^{b k g d}$ is the number of background events passing the selection criteria, $L$ is the integrated luminosity, $\epsilon_{e \mu}$ is the efficiency to pass an electron-muon-selection cut and $C_{b}$ is the correlation coefficient for the $b$-tagging algorithm. They all have been extracted from simulated samples, background estimations and additional luminosity measurements. From a unique solution of these linear equations, $\sigma_{t \bar{t}}$ and $\varepsilon_{b}$ can be directly calculated whereby the former one is the central aim of the analysis. The direct extraction of the tagger efficiency reduces the global uncertainties largely. The final results for the two parameters are [3]:

$$
\begin{array}{lll}
\sigma_{t \bar{t}}=182.9 \pm 7.1 \mathrm{pb}, & \varepsilon_{b}=0.557 \pm 0.009 & \text { for } \sqrt{s}=7 \mathrm{TeV} \\
\sigma_{t \bar{t}}=242.4 \pm 10.3 \mathrm{pb}, & \varepsilon_{b}=0.540 \pm 0.006 & \text { for } \sqrt{s}=8 \mathrm{TeV}
\end{array}
$$

For the subsequent extraction of $m_{t}^{\text {pole }}$, the $\sigma_{t \bar{t}}$ parameter was fitted as proposed in [5] resulting in Figure 1. The two intersection points of lines (theoretical predictions) and circles (measurements) for $\sqrt{s}=7 \mathrm{TeV}$ and $\sqrt{s}=8 \mathrm{TeV}$ lead to the value of $m_{t}^{\text {pole }}$. In the analysis, the extraction of the pole mass was instead performed by maximising the Bayesian likelihood

$$
\mathcal{L}\left(m_{t}^{\text {pole }}\right)=\int G\left(\sigma_{t \bar{t}}^{\prime} \mid \sigma_{t \bar{t}}\left(m_{t}^{\text {pole }}\right), \rho_{\text {exp }}\right) \cdot G\left(\sigma_{t \bar{t}}^{\prime} \mid \sigma_{t \bar{t}}^{\text {theo }}\left(m_{t}^{\text {pole }}\right), \rho_{\text {theo }}^{ \pm}\right) d \sigma_{t \bar{t}}^{\prime},
$$




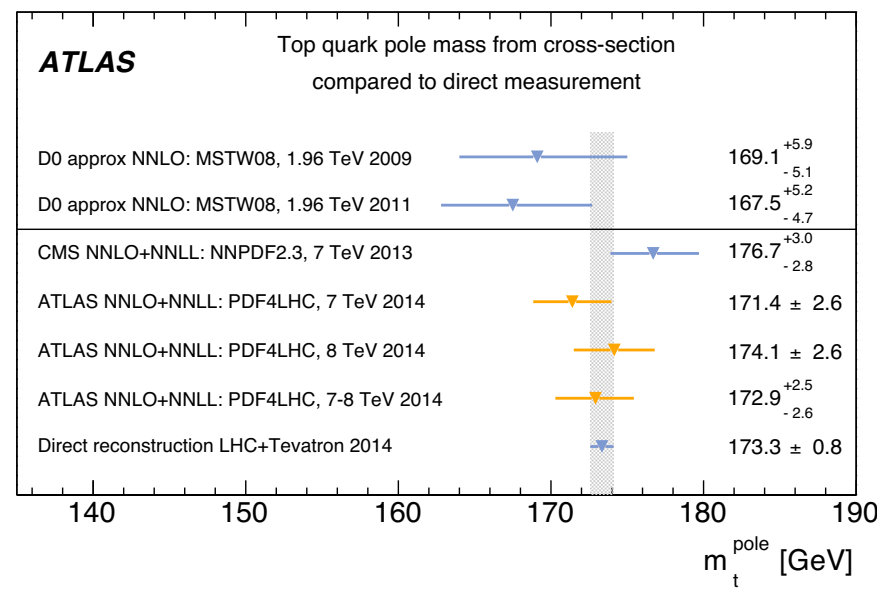

Figure 2: Overview of all recent top pole mass results. The presented analysis is listed in the penultimate row. For comparison, the top mass world average, a direct $m_{t}$ measurement of LHC+Tevatron from 2014 [6], can be found at the end [3].

resulting in a pole mass of

$$
m_{t}^{\text {pole }}=172.9_{-2.6}^{+2.5} \mathrm{GeV}
$$

for a combined measurement of the $\sqrt{s}=7 \mathrm{TeV}$ and $8 \mathrm{TeV}$ data. The dominant systematic uncertainties are the signal modelling of $t \bar{t}$ Monte Carlo (MC) events and the parton distribution functions (PDFs). The chosen top mass in the MC simulations has only a negligible effect on the final results of $\sigma_{t \bar{t}}$ and $m_{t}^{\text {pole }}$. A comparison to previous top pole mass measurements and to the 2014 world average $m_{\text {top }}$ measurement is shown in Figure 2. Additional information and all results presented here can be found in [3].

\section{First world combination of top-quark mass measurements}

A combined analysis of four experiments (ATLAS, CDF, CMS and D0) has been performed to determine $m_{\text {top }}$ at high precision [6]. The CDF and D0 collaborations used data from Tevatron Run II with an integrated luminosity of up to $8.7 \mathrm{fb}^{-1}$ with proton-antiproton collisions at $\sqrt{s}=1.96 \mathrm{TeV}$. The ATLAS and CMS collaborations used an integrated luminosity of up to $4.9 \mathrm{fb}^{-1}$ with proton-proton collisions at $\sqrt{s}=7 \mathrm{TeV}$ recorded at the LHC in 2011. The combination relied on the lepton+jets channels $(t \bar{t} \rightarrow \bar{l} v b q \bar{q} \bar{b})$ of all 4 experiments, the di-leptonic channels $(t \bar{t} \rightarrow \bar{l} v b l \bar{v} \bar{b})$ of all 4 experiments, the all-jets channel $(t \bar{t} \rightarrow q \bar{q} b q \bar{q} \bar{b})$ of CDF and CMS as well as the $E_{T}^{\text {miss }}$ channel from CDF, which is similar to the lepton+jets channel but uses the missing transverse energy $E_{T}^{\text {miss }}$ instead of lepton properties as a selection criteria. An overview can be found in Figure 3. The ATLAS measurements used template methods in 3D (lepton+jets) and 1D (di-leptonic). A combination of the 11 channels yields a top mass value of

$$
m_{t}[\mathrm{GeV}]=173.34 \pm 0.27 \text { (stat) } \pm 0.71 \text { (syst) } \quad \text { [6] }
$$




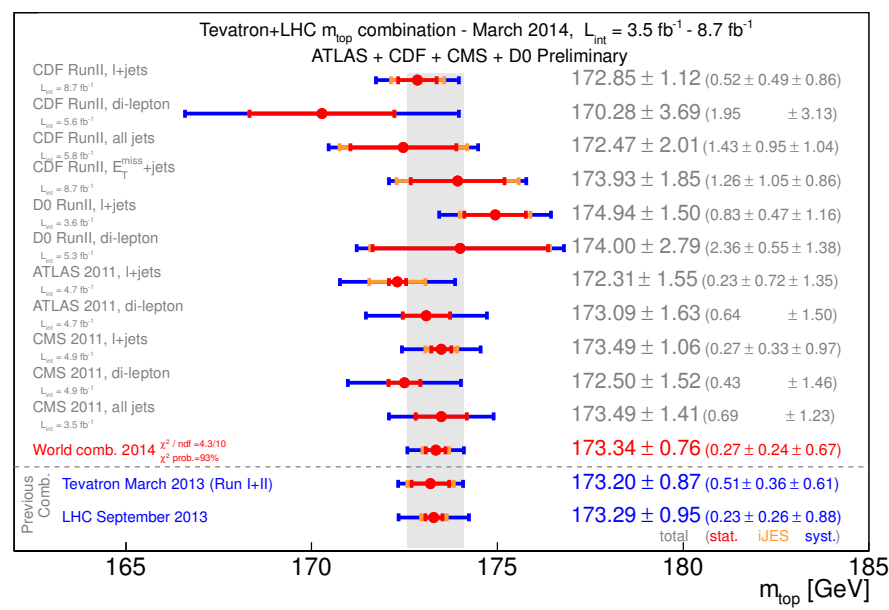

Figure 3: All 11 measurements from the Tevatron and the LHC used for the top mass world combination 2014 [6].

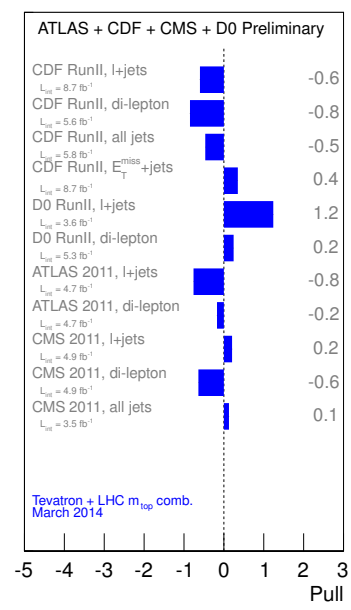

Figure 4: Pulls from the BLUE method for all inputs [6].

The hereby applied "Best Linear Unbiased Estimate" (BLUE) method [7, 8] determined the individual weights for the combination by minimising the total uncertainty of the final result. Compared to former single measurements, this analysis achieved a precision improvement of approximately $28 \%$ [9], and approximately $13 \%$ relative to the previous combination of the Tevatron [10]. The most dominant systematic uncertainties arise from the theory predictions in the $t \bar{t}$-MC sample and the models describing the responses of jets and $b$-tagged jets. All input measurements correlate well that results in the small pulls illustrated in Figure 4.

Apart from the combination itself the correlation assumptions were tested intensively but no strong dependency on the result was seen. To make future combinations easier several treatments of systematic uncertainty approaches were adopted and harmonised by the experiments.

\section{Measurement of the mass difference between top and anti-top quarks}

The CPT symmetry predicts equal masses for particles and anti-particles, a deviation would imply a non-local field theory in particle physics. The analysis [11] aimed at determining a possible mass difference between top and anti-top quarks by reconstructing the involved particles and their masses separately. This was done using an integrated luminosity of $4.7 \mathrm{fb}^{-1}$ recorded at the ATLAS detector in 2011 with $\sqrt{s}=7 \mathrm{TeV}$ proton-proton collisions. Each $t \bar{t}$ event in the lepton+jets channel was reconstructed with a kinematic $\chi^{2}$ fitter method while the top/anti-top mass difference was set as free parameter. Afterwards, the event topology was fixed by taking the smallest $\chi^{2}$ value per event, ending with the top/anti-top mass difference

$$
\Delta_{m(t, \bar{t})}^{f i t}=q_{l}\left(m_{b l v}^{f i t}-m_{b j j}^{f i t}\right)
$$

Different examples simulated for $\Delta_{m(t, \bar{t})}[\mathrm{GeV}]=-15,-5,0,5,15$ are shown in Figure 5. 


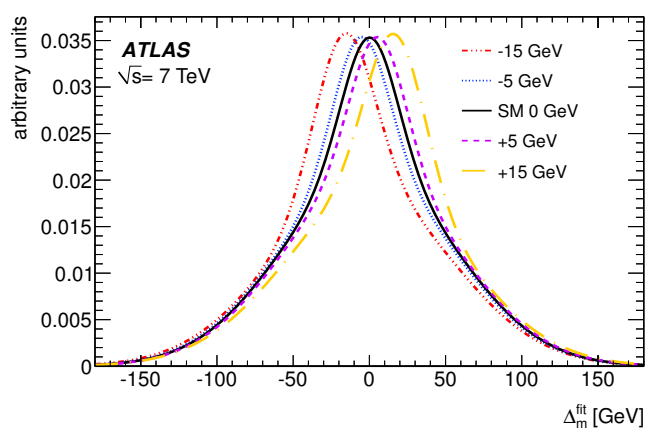

Figure 5: Simulation of several top/anti-top mass differences resulting in different templates for the describing parameter $\Delta_{m(t, \bar{t})}^{f i t} \quad[11]$.

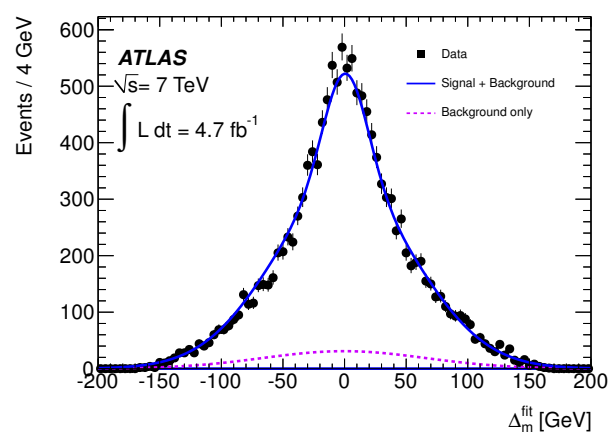

Figure 6: Maximum likelihood fit by signal and background templates for describing the data. Fit: $\chi^{2} /$ d.f. $=1.2 \quad[11]$.

Finally, the data distribution was fitted by templates with the sum of two Gaussians, representing the correct and incorrect jet-parton pairings in the $t \bar{t}$ reconstruction. The final maximum likelihood fit shown in Figure 6 results in a mass difference of

$$
\Delta_{m(t, \bar{t})}^{f i t}[\mathrm{GeV}]=0.67 \pm 0.61(\mathrm{stat}) \pm 0.41 \text { (syst) }
$$

which covers the SM prediction value and is therefore in agreement with the CPT invariance. In addition, the number of signal and background events $\left(n_{\text {sig }}, n_{b k g d}\right)$ could be extracted by the fit, being also consistent with the expectations. The systematic uncertainty of this measurement is dominated by the $b$ fragmentation model, which strongly influences the different detector responses of $b$ - and $\bar{b}$ quarks, resulting in an uncertainty of $\Delta_{b}(\Delta m)=0.34 \mathrm{GeV}$. All other uncertainties are relatively small with $\Delta(\Delta m)<0.10 \mathrm{GeV}$.

\section{Spin correlation in top/anti-top quark events}

The SM predicts a correlation between the spin polarisations of top and anti-top quarks thus the spin orientations are more often identical than opposite. Due to the absence of hadronisation, top quarks transfer their spin properties directly to the daughter particles and, e.g., affect the resulting angular distributions which can be examined, as published in [12]. The strength of the top spin correlation can be parametrised by

$$
A=\frac{N_{\text {parallel }}-N_{\text {antiparallel }}}{N_{\text {parallel }}+N_{\text {antiparallel }}}
$$

but depends on the quantisation basis. A typical value in the $t \bar{t}$ rest frame and with respect to the top quark flight direction was predicted to be $A_{\text {helicity, } S M}=0.31$ [13]. For a better comparison, the following results are represented by the coefficient $f_{S M}$ which is related to the number of spincorrelated $t \bar{t}$-events vs the total number of $t \bar{t}$-events. It is defined with the SM value $f_{S M}=1$. The data were acquired with the ATLAS detector in 2011 with $\sqrt{s}=7 \mathrm{TeV}$ and an integrated luminosity of 4.6 $\mathrm{fb}^{-1}$. Two decay channels have been used in this analysis: 


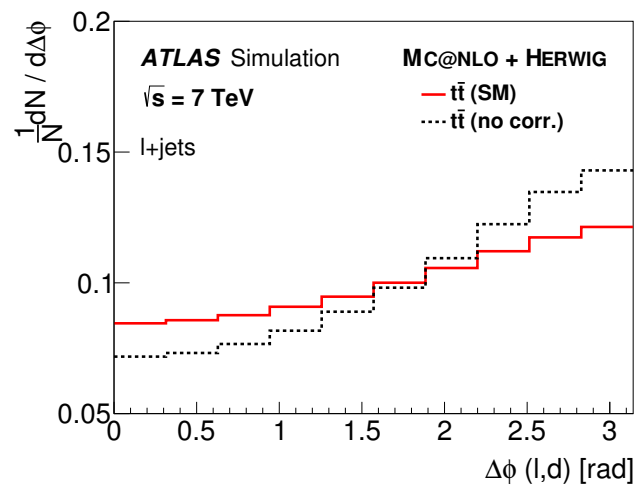

(a)

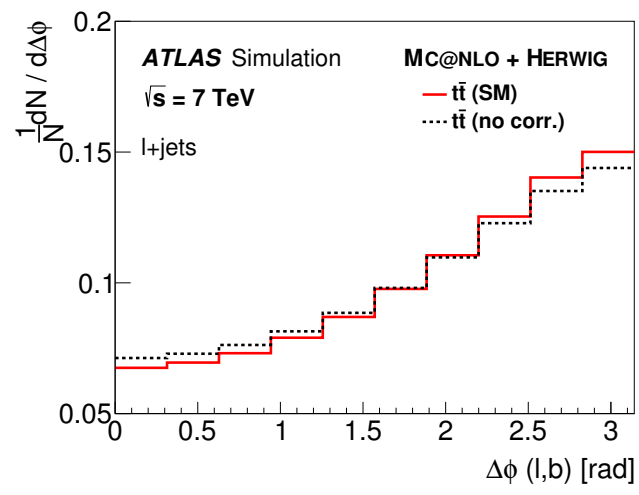

(b)

Figure 7: Discrimination variables for the spin correlation on parton level in the lepton+jets channel. The normalised distributions show the SM prediction in red solid lines and results for zero correlation in black dotted lines: (a) $\Delta \phi$ between lepton and light quark; (b) $\Delta \phi$ between lepton and $b$-quark [12].

- Events in the lepton+jets channel were reconstructed with the "Kinematic Likelihood Fitter" (KLFitter) algorithm [14]. Two observables were found to be sensitive to the differences between SM prediction and zero spin correlation, pictured in Figure 7 on parton level.

- Events in the di-leptonic channel were reconstructed with the help of the " $v$ weighting technique", based on a parameter scan over all pseudo-rapidities $\eta_{1}$ and $\eta_{2}$ of the two neutrinos involved. Four observables were found to be sensitive which are shown in Figure 8 on parton level.

To achieve the final spin correlation, templates were constructed for each signal and background sample separately. Maximum likelihood fits were performed afterwards, for a description of the data. The lepton+jets observables were combined into one result by a simultaneous fit to $\Delta \phi(l, d)$ and $\Delta \phi(l, b)$, while the four observables from the di-leptonic channel were fitted individually. The resulting values are summarised in Figure 9 while all are consistent with the SM prediction $f_{S M}=1$.

The observables $\Delta \phi$ (di-lepton), $\Delta \phi$ (lepton+jets) and S-ratio only allow for an indirect extraction of the spin correlation with respect to the SM, assuming the $t \bar{t}$ sample to be SM like. In contrary, the two $\cos \left(\phi_{\text {top }}\right) \times \cos \left(\phi_{\text {anti-top }}\right)$ observables describe a direct extraction, being sensitive also to contributions from physics beyond the SM.

The systematic uncertainties are widely spread while the most dominant arise from renormalisation/ factorisation scale and initial-/final-state-radiation models. The $\cos \left(\phi_{\text {top }}\right) \times \cos \left(\phi_{\text {anti-top }}\right)$ observables have three additional important uncertainties: the jet energy scale, the colour reconnection models and the low MC statistics. The $\Delta \phi(l, l)$ measurement instead has an additional strong dependence arising from $p_{T, t o p}$ reweighting.

\section{Top quark pair charge asymmetry measurements}

\subsection{Motivation and theoretical introduction}

Charge asymmetry measurements in $t \bar{t}$ events are an important test of higher order QCD, deviations from the theoretical predictions could point to new physics. Recent results from CDF and D0 indicated a significantly larger asymmetry than predicted by the SM [15, 16], while the latest LHC 


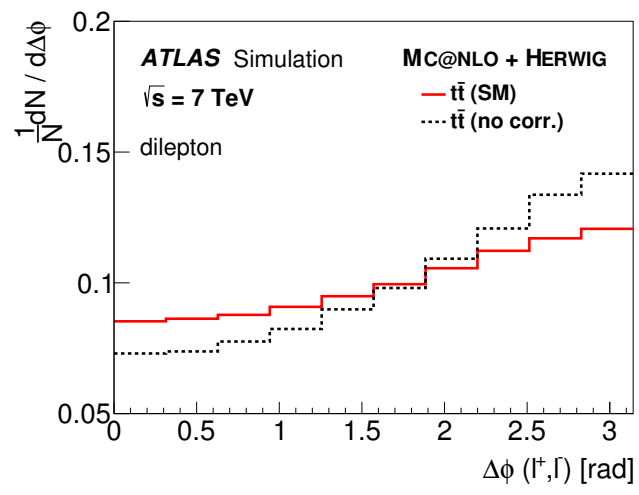

(a)

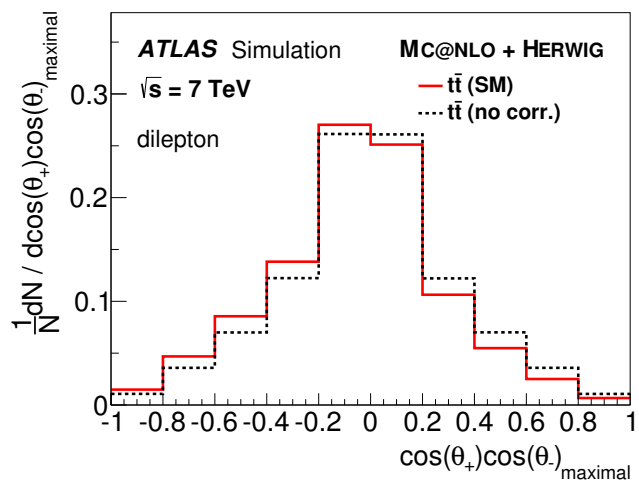

(c)

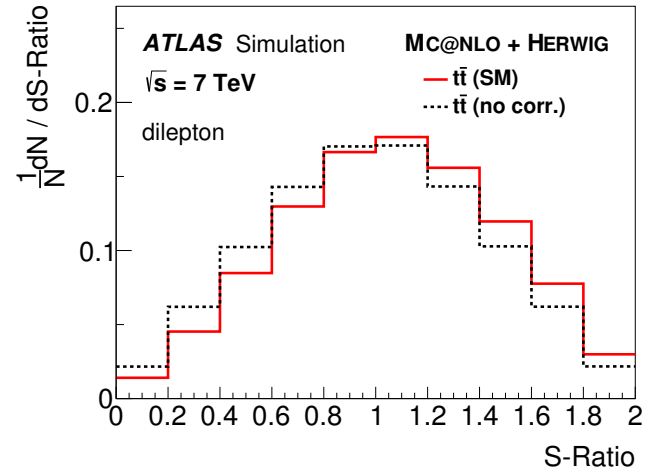

(b)

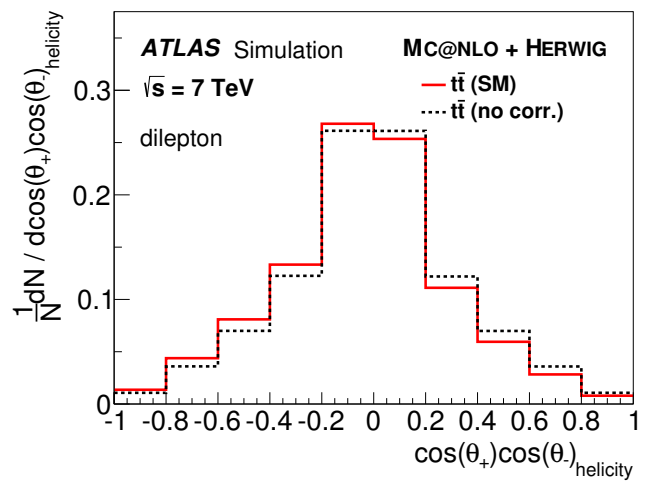

(d)

Figure 8: Discrimination variables for the spin correlation on parton level in the di-lepton channel. The normalised distributions show the SM prediction in red solid lines and results for zero correlation in black dotted lines: (a) $\Delta \phi$ between the two leptons; (b) "S-ratio": Specific ratio of spin correlated and uncorrelated matrix elements $\mathcal{M}$ describing the production and the decay of $t \bar{t}$ events, produced by like-helicity gluons $g_{R} g_{R}+g_{L} g_{L} \rightarrow t \bar{t}$; (c) $\cos \left(\phi_{\text {top }}\right) \times \cos \left(\phi_{\text {anti-top }}\right)$ in a basis maximising the spin correlation for each event separately; (d) $\cos \left(\phi_{\text {top }}\right) \times \cos \left(\phi_{\text {anti-top }}\right)$ in the $t \bar{t}$ rest frame pointing in the top quark direction [12].

measurements $[17,18]$ are compatible with the theoretical predictions of the SM but also with the deviation seen at Tevatron. These circumstances ask for an updated LHC measurement with higher precision.

Unlike the proton-antiproton collisions at Tevatron, the LHC is a proton-proton collider which admits no excess in the forward-backward-asymmetry $A_{F B}$. Instead, top quarks have a higher absolute rapidity and anti-top quarks occur more centrally, which is illustrated in Figure 10 (adopted from [19]) and in the following formula for the charge asymmetry $A_{C}$, with $\Delta|y|=\left|y_{\text {top }}\right|-\left|y_{\text {anti-top }}\right|$ : 


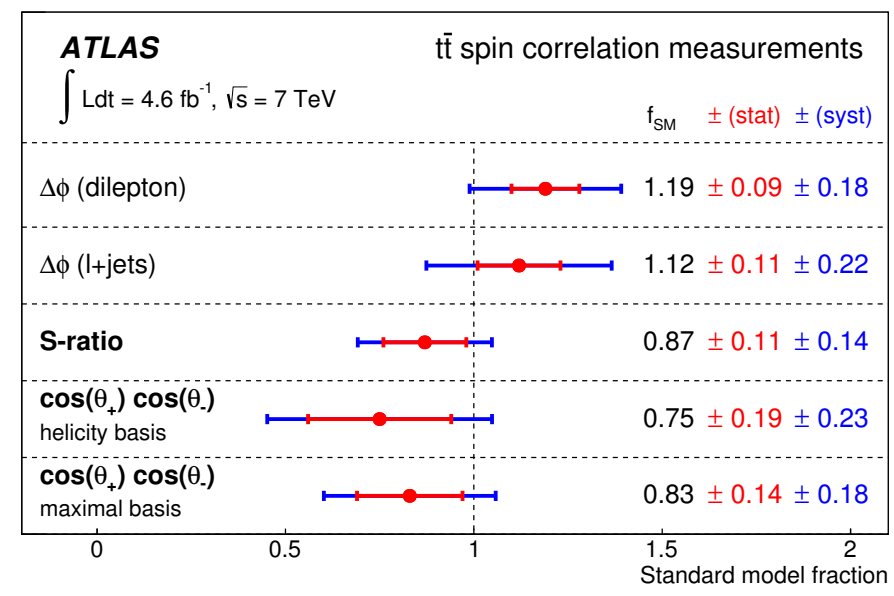

Figure 9: Summary of the separate spin correlation measurements. Consistency with the theoretical prediction is given for the SM fraction $f_{S M}=1$. Detailed explanations of the observables can be found in the text and in [12].

$$
A_{C}=\frac{N(\Delta|y|>0)-N(\Delta|y|<0)}{N(\Delta|y|>0)+N(\Delta|y|<0)}
$$
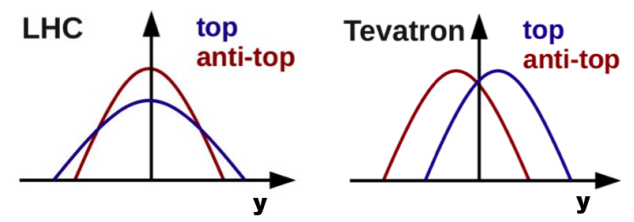

Figure 10: $t \bar{t}$ charge asymmetry comparison for LHC and Tevatron, adopted from [19].

\subsection{Measurement of the $t \bar{t}$ production charge asymmetry with the ATLAS detector}

The charge asymmetry measurement at ATLAS [20] was performed with $t \bar{t}$ events reconstructed in the lepton+jets channel. A $\sqrt{s}=7 \mathrm{TeV}$ dataset was used with an integrated luminosity of $4.7 \mathrm{fb}^{-1}$. By using kinematic fits, each top and anti-top 4-vector was reconstructed separately as well as the corresponding observable $\Delta|y|$. In addition, a "Fully Bayesian Unfolding" [21] was performed estimating $\Delta|y|$ at parton level. The resulting charge asymmetry observable $A_{C}$ was plotted as a function of $p_{T, t \bar{t}},\left|y_{t i t}\right|, m_{t \bar{t}}, m_{t \bar{t}, \beta_{z, t i t}>0.6}$ in Figure 11, including theoretical predictions for the SM as well as predictions for additional axigluons with masses of $300 \mathrm{GeV}$ and $7000 \mathrm{GeV}$. A summary of the resulting values can be found in Table 1 whereby the uncertainties are clearly dominated by statistics. Less than $10 \%$ of the total uncertainties arise from systematic variations.

For a better comparison, the plots in Figure 12 illustrate the forward-backward asymmetry measurements $A_{F B}$ from Tevatron and the charge asymmetry measurements $A_{C}$ from the LHC, including the here presented ATLAS results. 


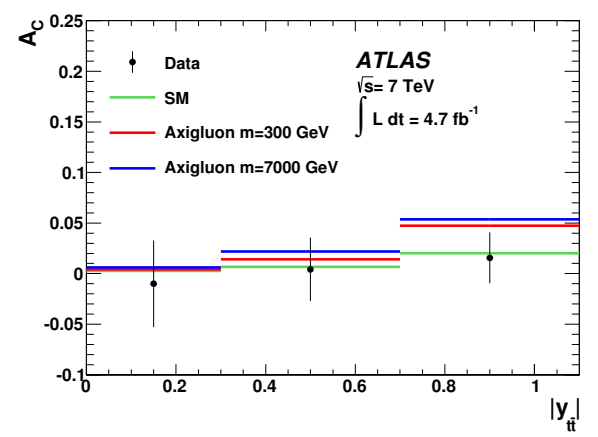

(a)

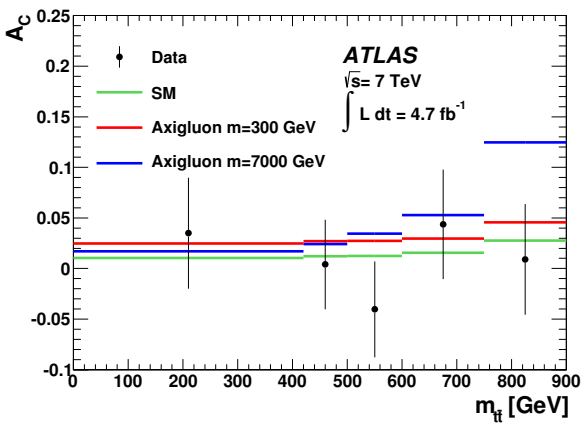

(c)

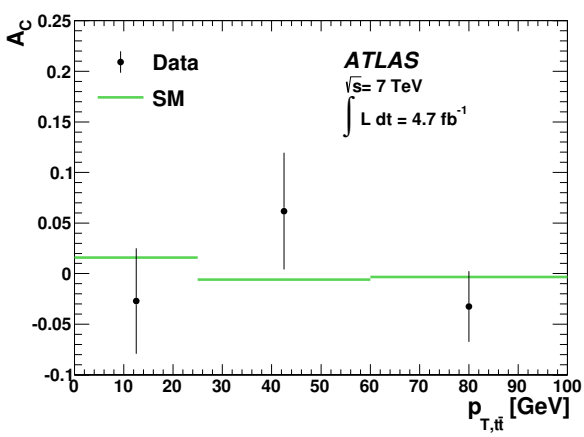

(b)

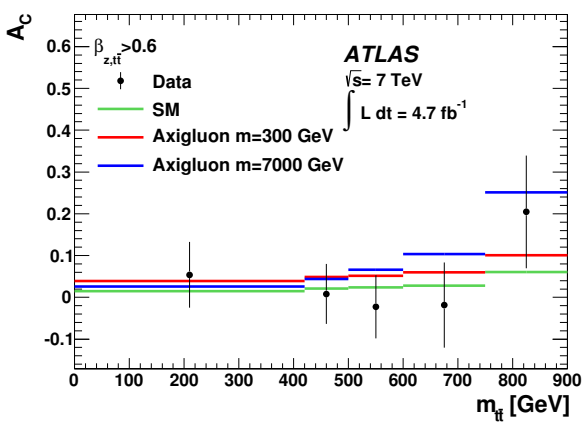

(d)

Figure 11: Charge asymmetry $A_{C}$ measurements. SM predictions are shown in green lines, predictions for additional axigluons $\left(\mathrm{m}=300 \mathrm{GeV}\right.$ and $7000 \mathrm{GeV}$ ) in red and blue lines: (a) $A_{C}$ as a function of the absolute $t \bar{t}$-pseudo-rapidity; (b) $A_{C}$ as a function of the transverse momentum of $t \bar{t}$; (c) $A_{C}$ as a function of the invariant mass of the top-pair-system; (d) Same as Figure (c) but for a boosted $t \bar{t}$ system expecting an enhanced effect on the detection of particles beyond the SM [12].

\begin{tabular}{l|l|l}
$A_{C}$ & Data (stat $\oplus$ syst) & Theory \\
\hline Unfolded & $0.006 \pm 0.010$ & $0.0123 \pm 0.0005$ \\
with $m_{t \bar{t}}>600 \mathrm{GeV}$ & $0.018 \pm 0.022$ & $0.0175 \pm 0.0005$ \\
with $\beta_{z, t \bar{t}}>0.6$ & $0.011 \pm 0.018$ & $0.020 \pm 0.007$
\end{tabular}

Table 1: Summary of the results which are all compatible with the theoretical predictions [20]. 


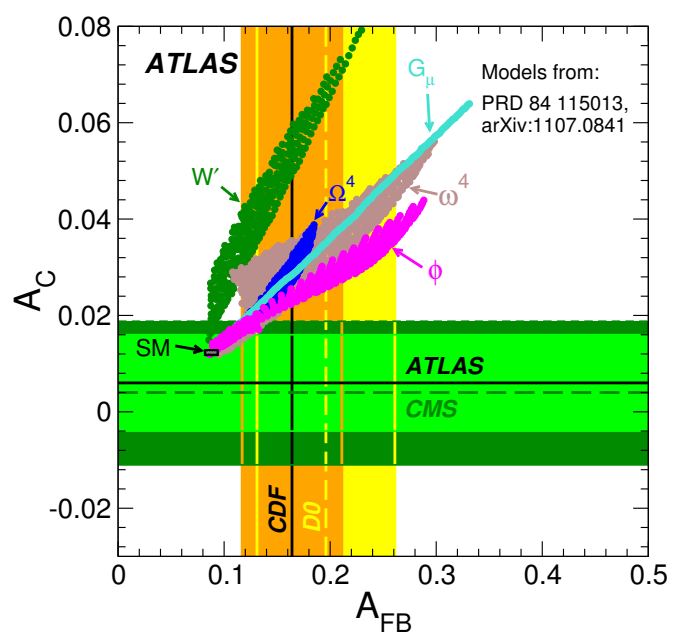

(a)

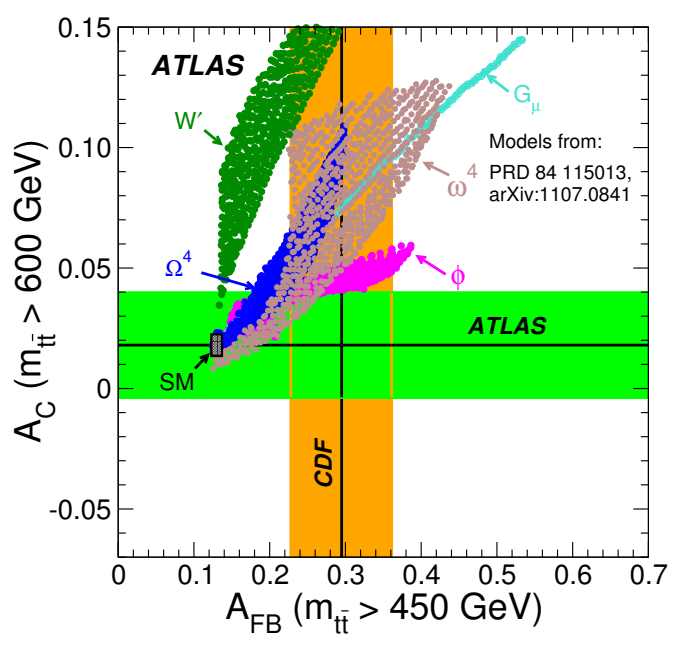

(b)

Figure 12: Measured $A_{F B}$ results from Tevatron and $A_{C}$ results from the LHC. The SM prediction is illustrated as a small black box while areas of potential new physics are drawn in pink $(\phi)$, dark green (W'), blue $\left(\Omega^{4}\right)$, brown $\left(\omega^{4}\right)$ and cyan $\left(\mathrm{G}_{\mu}\right)$. The right plot shows the measurements for boosted $t \bar{t}$-systems having an invariant mass $m_{t \bar{t}}>450 \mathrm{GeV}$ for the $A_{F B}$ measurement and an invariant mass $m_{t \bar{t}}>600 \mathrm{GeV}$ for the $A_{C}$ measurement. The LHC results are compatible with the SM prediction [20].

\subsection{Combination of ATLAS and CMS $t \bar{t}$ charge asymmetry measurements}

The analysis [22] combines two individual charge asymmetry measurements from ATLAS [20] and CMS [23] using an integrated luminosity of $4.7 \mathrm{fb}^{-1}$ and $5.0 \mathrm{fb}^{-1}$, respectively. All $t \bar{t}$ events have been reconstructed in the lepton+jets channel, while the combination itself was performed with the BLUE method, similar to the top mass world combination presented in Section 3. The final value of the combined top-pair charge asymmetry $A_{C}$ results into

$$
A_{C}=0.005 \pm 0.009 \text { (stat } \oplus \text { syst) } \quad \text { [22]. }
$$

This is compatible with the theoretical prediction of the SM: $A_{C}^{S M}=0.0115 \pm 0.0006$. Compared to the individual measurements an uncertainty improvement of $40 \%$ for CMS and $18 \%$ for ATLAS was achieved. The largest single systematic variation is the detector response model with an uncertainty of 0.004 .

Several stability tests have been performed by varying the correlation factors but no large effect on the final result was observed. In addition, the pulls of the two input measurements were found to be relatively small with

$$
\frac{A_{A T L A S}-A_{c}}{\sqrt{\sigma_{A T L A S}^{2}-\sigma_{A c}^{2}}}=+0.109 \quad \text { and } \quad \frac{A_{C M S}-A_{c}}{\sqrt{\sigma_{C M S}^{2}-\sigma_{A c}^{2}}}=-0.109 \text {. }
$$

An overview of the final result, the input for the combination as well as the theoretical prediction are visualized in Figure 13. 


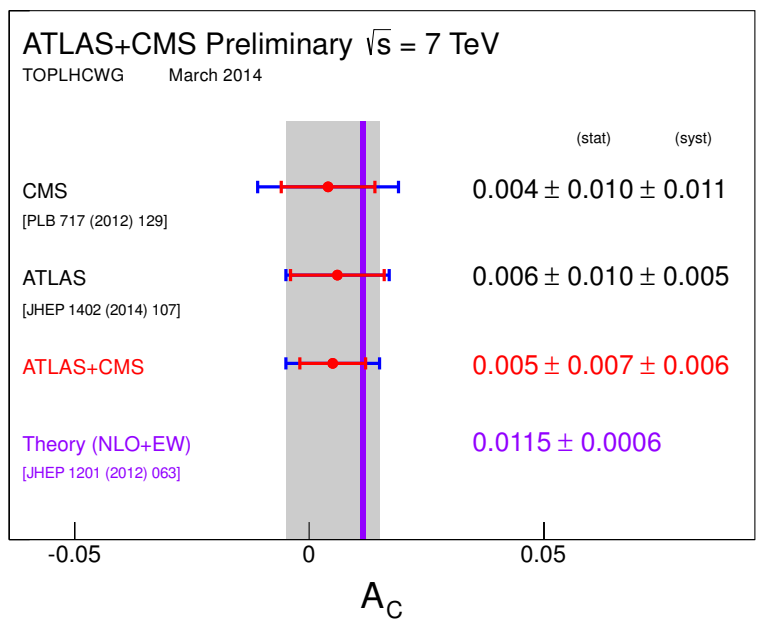

Figure 13: Summary of the combined result for the charge asymmetry $A_{C}$ measurement of ATLAS and CMS. In addition, the individual measurements and the SM prediction are shown. Statistical uncertainties are drawn in red, the total uncertainties in blue [22].

\section{Conclusions}

A selection of the most recent top mass and top properties measurements from the ATLAS detector were presented. The topics covered included $t \bar{t}$ spin correlation, $t \bar{t}$ charge asymmetry, top/anti-top mass difference and the top mass itself. The large number of reconstructed top quarks allowed in all cases high precision measurements and significant Standard Model tests. A great reduction of uncertainties has been achieved compared to former measurements. Each result is in very good agreement with the SM and no hints for physics beyond the SM were detected.

\section{References}

[1] K.A. Olive et al. (Particle Data Group), Chinese Physics C 38, 090001 (2014)

[2] ATLAS collaboration, JINST 3, S08003 (2008)

[3] ATLAS collaboration, arXiv:1406.5375 (2014)

[4] D0 collaboration, V. M. Abazov et al., Phys. Rev. D 84, 032004 (2011)

[5] M. Czakon, P. Fiedler and A. Mitov, Phys. Rev. Lett. 110, 252004 (2013)

[6] ATLAS, CDF, CMS and D0 collaborations, arXiv:1403.4427 (2014)

[7] L. Lyons, D. Gibaut and P. Clifford, Nucl. Instrum. Meth. A270, 110 (1988)

[8] A. Valessi, Nucl. Instrum. Meth. A500, 391 (2003)

[9] CMS collaboration, JHEP 12, 105 (2012)

[10] Tevatron Electroweak Working Group, arXiv:1305.3929 (2013)

[11] ATLAS collaboration, Phys. Lett. B 728C, 363-379 (2014)

[12] ATLAS collaboration, arXiv:1407.4314 (2014)

[13] W. Bernreuther and Z. -G. Si, arXiv:1305.2066 (2013)

[14] J. Erdmann et al., Nucl. Instr. and Meth. in Phys. Res. A 748, 18 (2014) 
[15] CDF collaboration, T. Aaltonen, et al., Phys. Rev. D 87, 092002 (2013)

[16] D0 collaboration, V. M. Abazov et al., Phys. Rev. D 84, 112005 (2011)

[17] CMS collaboration, Phys. Lett. B 717, 129 (2012)

[18] ATLAS collaboration, Eur. Phys. J. C 72, 2039 (2012)

[19] Yves Sirois, CMS Status Report, 110th LHCC meeting at CERN (2012), http://cds.cern.ch/record/1455678/

[20] ATLAS collaboration, JHEP 02, 107 (2014)

[21] G. Choudalakis, arXiv:1201.4612 (2012)

[22] ATLAS and CMS collaborations, ATLAS-CONF-2014-012 and CMS PAS TOP-14-006, http://cds.cern.ch/record/1670535/ and http://cds.cern.ch/record/1670517/

[23] CMS collaboration, JHEP 04, 191 (2014) 